\title{
Biocompatibility study of poly(vinyl alcohol)-based electrospun scaffold for hernia repair
}

\author{
K. Molnár ${ }^{1}$, C. Voniatis ${ }^{1,2}$, D. Fehér ${ }^{2}$, A. Ferencz ${ }^{2}$, L. Fónyad ${ }^{3}$, L. Reiniger ${ }^{3,4}$, M. Zrínyi ${ }^{1}$, \\ Gy. Wéber ${ }^{2}$, A. Jedlovszky-Hajdú ${ }^{1}$ \\ ${ }^{1}$ Laboratory of Nanochemistry, Department of Biophysics and Radiation Biology, Semmelweis University, Budapest, \\ Hungary \\ ${ }^{2}$ Department of Surgical Research and Techniques, Semmelweis University, Budapest, Hungary \\ ${ }^{3} 1^{\text {st }}$ Department of Pathology and Experimental Cancer Research, Semmelweis University, Budapest, Hungary \\ ${ }^{4}$ MTA-SE NAP, Brain Metastasis Research Group, Hungarian Academy of Sciences, $2^{\text {nd }}$ Department of Pathology, \\ Semmelweis University, Budapest, Hungary
}

Received 20 December 2017; accepted in revised form 28 February 2018

\begin{abstract}
Abdominal hernia is a purely surgical disorder where due to a defect in the abdominal wall, tissues or organs can extrude out of the abdominal cavity. The only conclusive treatment is surgical making a mesh implantation indispensable. Tissue engineering is now a promising method for creating scaffolds that provide an adequate support for tissue ingrowth. Our purpose was to develop a non-adhesive hernia mesh, which could be used in the repair of abdominal wall hernias but concurrently a scaffold for abdominal tissue regeneration. Poly(vinyl alcohol) bulk hydrogels are promising materials in wound dressing hence, interest in electrospun poly(vinyl alcohol) meshes has emerged in the past few years for different biomedical applications. In the present paper, preparation of electrospun poly(vinyl alcohol) fiber membranes and their in vitro and in vivo behaviors were followed to study the adhesion, biocompatibility, and biodegradability of the meshes. Our results showed that the surface of PVA meshes does not favor cell adhesion in vitro. During the animal experiments, PVA meshes demonstrated good integration into the surrounding tissue with minimal inflammatory reaction and minimal adhesions to intra abdominal structures.
\end{abstract}

Keywords: nanomaterials, PVA scaffold, electrospinning, hernia mesh, mesh incorporation

\section{Introduction}

Abdominal wall hernia is a special clinical problem, where the previously damaged abdominal wall is not able to fulfil its physiological functions and surgical reinforcement is mandatory. According to the literature, incidence of incisional hernias after laparotomy is about $11-20 \%[1,2]$. Although mesh implantation has improved the outcome significantly, the problem with open procedures is that they involve extensive dissection associated with a $20 \%$ wound complication rate requiring further surgery in many patients. Laparoscopic mesh implantation has gained acceptance among surgeons and patients by decreasing this high complication rate $[3,4]$. One drawback of this technique is that the prosthetic mesh should be placed intraperitoneal, directly adjacent to the intestines, and may cause life threatening complications (infection, fistula, etc.). Repair of damaged tissues or organs is a significant clinical problem, which can be treated using artificial scaffolds.

Resorbable synthetic meshes represent a logical improvement of non-resorbable meshes, however, as scaffolds to support native tissue ingrowth, they must be present until full regeneration of the damaged

${ }^{*}$ Corresponding author, e-mail: hajdu.angela@med.semmelweis-univ.hu (C) BME-PT 
tissue without any strength loss [5]. Although there are resorbable meshes with controlled degradation available on the market, their high price hinders their general use, whereas synthetic meshes are still limited by their loss of strength over time [6].

Tissue engineering is now a promising method for creating scaffolds that provide an adequate support for tissue ingrowth. Electrospinning is a frequently used technology to build artificial networks for tissue replacement. Preparation of thin polymer fibers for biomedical applications, which are biocompatible and biodegradable, is a very progressive research area nowadays. Nanofibers have all the necessary properties that are important to mimic the tissue in a living system. The fibers have high surface-volume ratio, the fiber mats are highly porous with interconnected pore structure, and it is easy to modify their surface functionality and mechanical properties (e.g., tensile strength, stiffness, etc.) in a wide range [7].

Poly(vinyl alcohol) (PVA) bulk hydrogels are promising materials in wound dressing, as it is known from the literature $[8,9]$. PVA is a non-toxic hydrophilic polymer, which is biocompatible and fairly biodegradable with lots of well-known biomedical applications (such as contact lenses, implants, and drug additives) $[10,11]$. Thus, PVA is an eligible choice for the preparation of meshes for tissue engineering [12]. Furthermore, the background of PVA mats prepared with electrospinning method has broadly developed in the past few years $[13,14]$. There were several attempts to use PVA also in hernia treatment [15], however, to the best of our knowledge, no PVA mesh that perfectly diminishes the side effects of mesh based hernia repair (such as adhesion, inflammation, etc.) has been prepared so far.

The aim of this study is to develop a non-adhesive absorbable hernia mesh, which can be used even in an intraperitoneal position and can act as a scaffold for the regeneration of the abdominal tissue, then disappear when the abdominal wall and scar tissue are strong enough. For this purpose, we evaluate the preparation of electrospun PVA fiber mats, their interaction in vitro with human lung carcinoma cell line (A549), but also in vivo with animal models, using laboratory rats to follow any adhesion formation, and to determine the biocompatibility and biodegradability of the meshes. Size and structure of fibers have been investigated with Scanning Electron Microscope.

\section{Materials and methods}

Poly(vinyl alcohol) (PVA) $\left(M_{\mathrm{w}} \sim 72000\right)$ was purchased from Merck-Scuchardt (Hohenbrunn, Germany), whereas glutaraldehyde was purchased from Merck (Darmstadt, Germany). Sodium-azide, minimal essential media (DMEM 6546) and phosphate based saline (PBS) were purchased from SigmaAldrich (USA), $\mathrm{HCl}$ from Reanal (Budapest, Hungary), $\mathrm{ClO}_{2}$ (Solumium) from Sanitaria (Budapest, Hungary), foetal calf serum from Kvalitex (Budapest, Hungary), L-glutamine and Vybrant Did from Thermo Fischer Scientific (Waltham, USA), gentamicin from Sandoz (Holzkirchen, Hungary), ciprobay (Ciprinol-KRKA, Hungary). Polypropylene mesh (Monofilament Knitted Polypropylene mesh) was purchased from Bard Davol Inc. (Warwick, USA), 4-0 Polydioxanone, monofilament suture line from Atramat (Mexico City, Mexico), polydioxanone 5-0 sutures (PDS) and polypropylene 5-0 sutures (Prolene) from Ethicon Inc. (Somerville, NJ, USA).

\subsection{Preparation of the polymer solution}

Poly(vinyl alcohol) was dissolved in boiling distilled water under strong stirring, in order to create a concentrated solution. The final PVA concentration was determined gravimetrically from samples dried at $60^{\circ} \mathrm{C}$; then the raw solution was diluted to the desired concentration ( $8 \mathrm{~g}$ polymer in $100 \mathrm{~g}$ solution). A small amount of sodium-azide was added to stock suspension to avoid infection.

\subsection{Electrospinning technique}

A custom electrospinning setup was used to create thin polymer fibers from PVA [16]. The mixture of $3.25 \mathrm{~mL} 8 \mathrm{w} \%$ PVA and $62.5 \mu \mathrm{L}$ of glutaraldehyde (GDA) solution was delivered by a syringe pump (KD Scientific KDS100, Holliston, USA) with a glass syringe (Fortuna Optima 7.140-33, Sigma-Aldrich, USA) with a metal Hamilton tip at a feeding rate of $1 \mathrm{~mL} / \mathrm{h}$. The electrode was attached to the metal tip and a high voltage (16-20 kV) was applied during sample production using a high voltage DC power supply (Genvolt 73030P, Bridgnorth, UK). We used common aluminium foil as target placed $15 \mathrm{~cm}$ in front of the needle.

\subsection{Microscopic techniques}

For the determination of the morphology and average diameter of PVA fibers, Scanning Electron 
Microscopy (SEM) was used. Micrographs were taken using a ZEISS EVO 40 XVP (Carl Zeiss AG, Oberkochen, Germany), equipped with an Oxford INCA $\mathrm{X}$-ray spectrometer (EDS) (Oxford Instruments, Abingdon, UK). An accelerating voltage of $125 \mathrm{kV}$ was applied; the actual voltage was $20 \mathrm{kV}$ in each case. Samples were fixed on a special conductive sticker with tweezers. For the measurements, samples were sputter coated with gold in 20-30 nm thickness with a 2SPI Sputter Coating System (West Chester, USA). In the case of cross-linked PVA membrane, it was washed with ultrapure water and freeze-dried. The average fiber diameter was determined by measuring 50 individual fibers.

\subsection{Formulation and cross-linking of PVA meshes for mechanical, in vitro and in vivo experiments}

For handling and suturing during surgery, a single sheet of PVA mesh is not suitable. Therefore, to strengthen the meshes, they were folded into approximately $5 \times 5$ or $4.5 \times 3.5 \mathrm{~cm}$ sized squares, and were pressed with a hydraulic press (Atlas Manual 15T (Orpington, UK), generally used for pastille formulation in infrared spectroscopy) to ensure the integrity of its layers. The samples were placed in a sandwich of aluminum foil, several layers of paper and wood pressure plates, then pressed by $5 \mathrm{t}$ on their whole surface for 5 minutes.

To stabilize the PVA based fiber mesh and prohibit its dissolution, we cross-linked the PVA polymer inside the fibers. The previously pressed fibrous sheets were immersed into $2 \mathrm{M} \mathrm{HCl}$ solution for 1 hour, and washed with ultrapure water until the supernatant became neutral. The samples were then stored in PBS with added chlorine dioxide for sterilization, and were incubated at $37^{\circ} \mathrm{C}$ for 1 week $[17,18]$.

\subsection{Cell experiments}

A549 human lung carcinoma adherent epithelial-like monolayer cells (passage 13-22 after defrosting from liquid nitrogen) were used for the observation of any toxic effect of our meshes, caused by chemicals released from the samples or degradation byproducts. For these experiments, PVA scaffolds and its compounds, namely PVA solutions of different concentrations and GDA were used. PVA meshes were pretreated with $2 \mathrm{M} \mathrm{HCl}$ and sterilized with $\mathrm{ClO}_{2}$ (as described in Section 2.4). Our pilot tests also proved that $\mathrm{ClO}_{2}$ has no negative effects on the proliferation or viability of the cells, supporting the well-known antimycotic and antibiotic effect of $\mathrm{ClO}_{2}[17,18]$. Electrospun PVA scaffolds were washed with ultrapure water before the cell experiments. The scaffolds were tested after washing (Method A) and after incubating in cell medium for $72 \mathrm{~h}$ (Method B).

For the experiments, cells were cultivated in minimal essential medium supplemented with $10 \%$ foetal calf serum, 4\% L-glutamine and Gentamicin. $2.5 \cdot 10^{5}$ cells/ Petri dish were spread for each experiments. All of the samples were incubated at humidified incubator $\left(37^{\circ} \mathrm{C} ; 5 \% \mathrm{CO}_{2}\right)$. The cells were treated with Ciprobay $(25 \mu \mathrm{L} / 5 \mathrm{~mL})$ to prevent any infection.

For the consecutive experiments, PVA solutions of different concentrations in the range of $10^{-8}$ to $0.8 \mathrm{~g} / 100 \mathrm{~g}$ (9 defined concentrations), GDA solution, and a PVA solution made from the supernatant of a 72 hour medium incubated PVA mesh were added to the cell media before seeding the cells.

All experiments were conducted with 3 parallel measurements, using a control cell population without any treatment. For tracking proliferation on the scaffolds, cells were labelled with Vybrant Did solution. These experiments were carried out in 24 well/plates. Before seeding, cells were incubated in $100 \mu \mathrm{L}$ of staining medium for 15 minutes. The staining medium was prepared by adding $5 \mu \mathrm{L}$ of the supplied dye labelling solution to $1 \mathrm{~mL}$ of serum free growth medium. After 15 minutes of incubation, the staining medium was dripped off, and the culturing flask was washed three times with normal growth medium before incubating it again for 10 minutes. Cells were removed by using a trypsin- EDTA solution. The labelled cells were seeded into a 24 well plate (Sarstedt AG \& Co. KG, Nümbrecht, Germany) at a $3 \cdot 10^{4}$ cells $/ \mathrm{mL} /$ well.

\subsection{Mechanical tests}

Simple mechanical tests were carried out to predict the mechanical and surgical performance of the samples, where suturability and maximum strength against pulling forces were investigated. The tearing point of the PVA mesh was measured by unidirectional extension in a ZWICK Z050 Elastic Modulus Meter (Ulm, Germany) at room temperature. For the measurement $15 \times 50 \mathrm{~mm}$ rectangles were cut and held by simple clamps. The measurement was carried out at $10 \mathrm{~mm} / \mathrm{min}$ cross-head speed in 3 parallel experiments.

A modified version of the simple running suture was used in order to recreate the state of a sutured hernia 

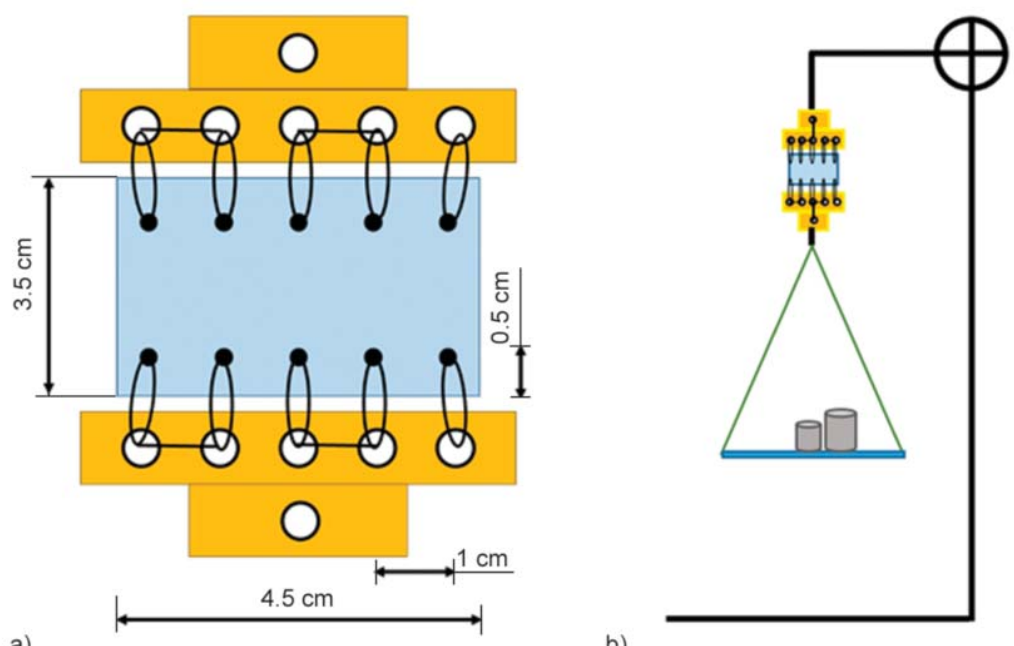

b)

Figure 1. Schematic representation of the modified simple running suture samples (a), schematic figure of measurement setup (b).

mesh after implantation but enables simple mechanical testing. Previously prepared and pressed PVA meshes (Section 2.4) and a commercially available polypropylene (PP) mesh were sutured with a 4-0Polydioxanone, monofilament suture line between two holders. The distance between the loops was kept at $1 \mathrm{~cm}$ (which refers to the standard procedure during a surgical mesh implantation), while the distance from the edge of the samples at $0.5 \mathrm{~cm}$, replicating the implanted samples. The schematic drawing of the suture with the sutured PVA or PP mesh can be seen on Figure 1a.

To test their strength and mechanical behaviour, our samples were hanged in a frame with a plate attached to their bottom. Different standard weights were successively placed on the plate until the samples were torn (Figure 1b).

\subsection{Animal studies}

The aim of the first series of experiments was to study the biological behaviour of intra-peritoneally implanted PVA meshes. Sixty inbred rats of Wistar strain, weighting 315-350 g, were used. They were bred under specific pathogen-free conditions, kept under standard laboratory conditions (temperature of $20-24^{\circ} \mathrm{C}$, relative humidity of $50-60 \%, 12 \mathrm{~h}$ light/ $12 \mathrm{~h}$ dark), fed with laboratory diet, and given water ad libitum. In all animals, a $4 \mathrm{~cm}$ left paramedian laparotomy incision was performed. The 60 rats were randomly divided into four groups.

In group I $(n=15)$, and secured to the abdominal wall by suturing the four corners using absorbable polydioxanone $5-0$ sutures.
In group II ( $n=15)$, a full-thickness abdominal wall defect $(2 \times 2 \mathrm{~cm})$ was created, and PVA mesh was fixed intraperitoneally with absorbable polydioxanone 5-0 sutures.

In group III ( $n=15)$, a similar abdominal wall defect was created, and the defect was repaired with a PVA mesh. This mesh was fixed intraperitoneally with running polypropylene 5-0 sutures (Figure 2).

In group IV (control group, $n=15$ ), rats underwent the same procedure as in group I, except there was no mesh implantation. In all animals the muscle and skin incision were closed with an 5-0 absorbable polydioxanone running suture.

Animals were housed in individual cages, and observed daily for evidence of wound complications,

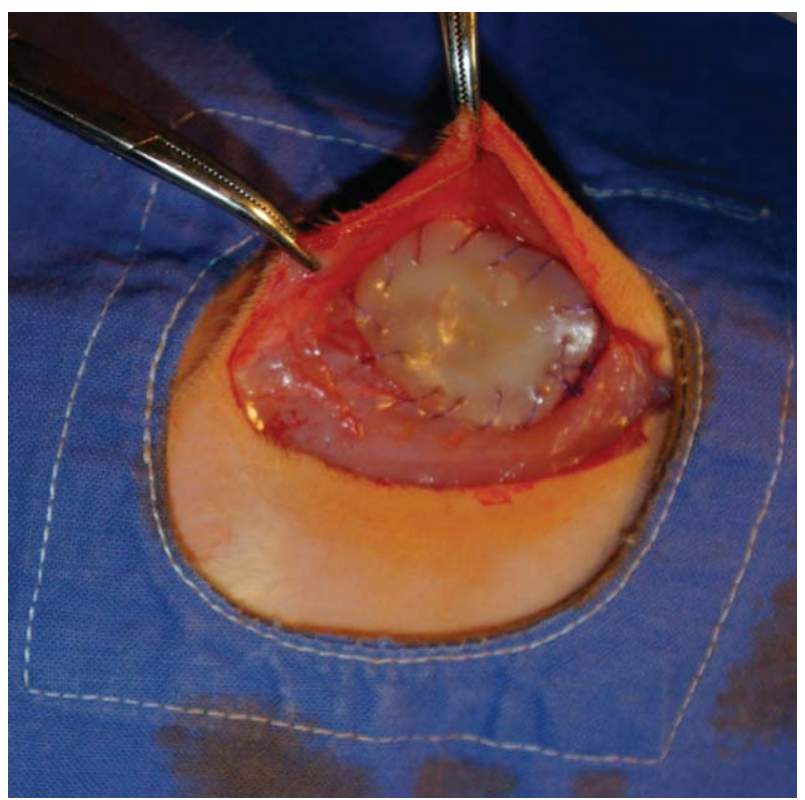

Figure 2. PVA mesh secured with running suture. 
such as infection, seroma, abscess, hematoma, or skin dehiscence. The experimental protocol adhered to rules laid down by the Directive of the European Parliament and of the Council on the protection of animals used for scientific purposes, and was approved by the Semmelweis University's Institutional Animal Care and Use Committee. The accreditation number of the laboratory is $22.1 / 1244 / 3 / 2011$.

Animals were terminated and the meshes were removed on the $7^{\text {th }}, 14^{\text {th }}, 28^{\text {th }}, 90^{\text {th }}, 178^{\text {th }}$ postoperative days to determine tissue ingrowths, and the degree of adhesion formations (macro- and microscopic appearance). We had to terminate three animals (one animal from group II, and two from group III) earlier as planned due to technical issues (inadequate fixation). The presence of adhesions was assessed by their intensity, surface area, and total score were assessed visually, using a modified Diamond scale [19].

\subsection{Histological evaluation}

Tissue samples were fixated in $10 \%$ buffered formalin and embedded in paraffin. $4 \mu \mathrm{m}$ sections were prepared then stained with Haematoxylin and Eosin. Glass slides were digitalized with a Panoramic 250 Flash Scanner (3DHISTECH Ltd., Budapest, Hungary).

\section{Results and discussion}

\subsection{Characterization of electrospun PVA fibers}

After a long optimization process (results are not shown here), homogenous nanofibers were created. During electrospinning the water evaporated from the system, and a non-woven dry and white mesh was produced (Figure 3a).

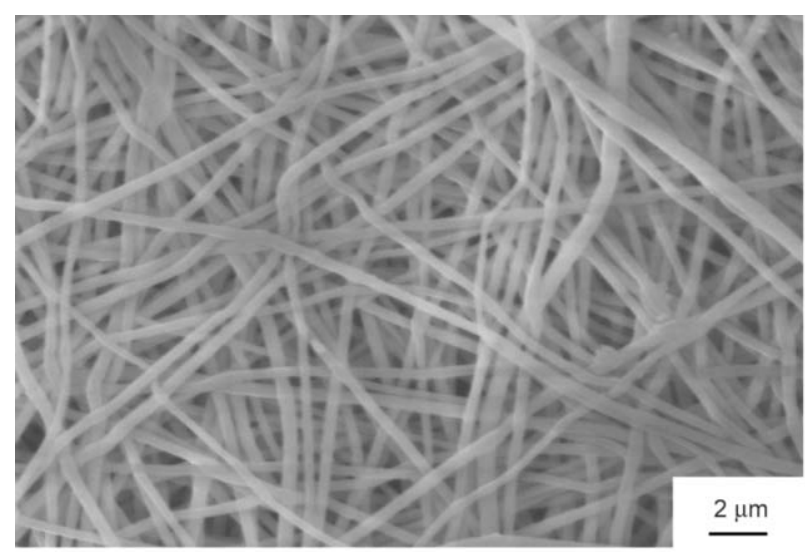

a)
According to SEM, electrospinning yielded smooth and fairly uniform fibers with an average diameter of $390 \pm 20 \mathrm{~nm}$. There were very few small spheres of polymer, which we originate from the stabilizing jet at the beginning of the spinning process. SEM also showed that the diameter and shape of the fibers can be reproduced in a well-controlled way.

Generally, this technique uses very toxic solvents during the fiber formation, such as dimethyl formamide, or trifluoroacetic acid [20], hence, in our case, the solvent was water. In this case, as an advantage of our setup, the residual solvent - which is not able to evaporate during the electrospinning - could not cause any side effect during the application.

\subsection{Formulation and cross-linking of PVA meshes for further experiments}

To prepare a required polymer matrix for the different experiments, such as cell, mechanical, and animal studies, having chemical cross-links between the polymer chains is essential. Since an implant for abdominal wall regeneration should withstand the abdominal pressure, it was necessary to strengthen our samples. Folding the electrospun meshes into suitable sized samples before further experiments (in vivo or mechanical) significantly increased their strength, however, it also reduced their flexibility, as we obtained a thick, paper-like sheet in each case. Furthermore, SEM showed that the fiber density in the pressed sample was raised without any observable damage of the fibrous structure (Figure 3a).

Acidic $\mathrm{pH}$ enables GDA to create cross-links between the PVA chains, therefore samples were immersed into $2 \mathrm{M} \mathrm{HCl}$ solution. As the paper-like sheets sucked the acidic solution, the white mesh

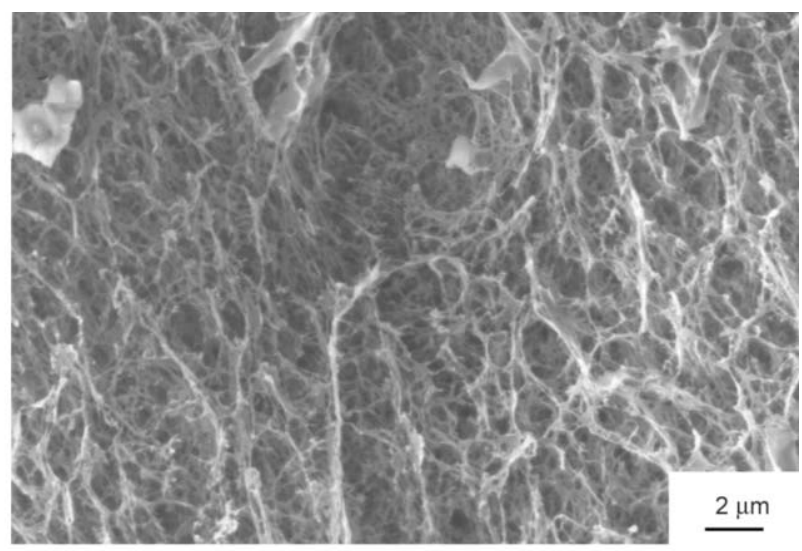

b)

Figure 3. Pictures taken of PVA membranes in different states: SEM picture taken of the fibrous structure after compression (a) and after chemical cross-linking (b). 
turned into opaque indicating that cross-links were formed. In order to prove the presence of cross-links, and thus the gelatic property of the fibers, a scaffold was stored in water at a temperature above $90^{\circ} \mathrm{C}$. Scaffolds without $\mathrm{HCl}$ pretreatment dissolved instantly, while pretreated ones did not, leading us to the conclusion that immersing the pressed samples into $\mathrm{HCl}$ does indeed induce cross-link formation. In order to see the microscopic structure of treated samples, the membrane was washed thoroughly, freeze-dried and investigated by SEM. As it can be seen on Figure $3 b$, the fibrous structure was damaged, as the fibers fused together, creating an interconnected spongelike structure. Fusion of fibers or gel fibers is a very common problem occurring in different degrees, especially in the case of PVA membranes [21-24]. However, the porous structure of our membranes still satisfies our objectives. After washing the samples with ultrapure water, they were stored in PBS for 1 week at $37^{\circ} \mathrm{C}$. As a result, rubber-like flexible and opaque gel sheets were obtained. These samples were used in further experiments.

\subsection{Interaction between cells and PVA, GDA and $\mathrm{ClO}_{2}$ solutions}

It is always a hard task to choose an appropriate cell line for basic in vitro tests. A549 is a well-known cancer cell line, frequently used for similar kind of applications, with a tremendous amount of information available in the literature related to the biocompatibility of electrospun meshes [25-27].

For biomedical applications, it is important to exclude side effect arising from building blocks. During metabolism in a living system, polymers typically break up into smaller fragments and monomers, thus, proving the non-toxic behaviour of the ingredients is inevitable. Therefore, the toxicity of the compounds of the PVA scaffolds was investigated.

The examination of PVA solutions $\left(10^{-8}\right.$ to $\left.0.8 \mathrm{~g} / 100 \mathrm{~g}\right)$, as the main building block in our matrix, resulted in normal, healthy triangular shaped cells after $24 \mathrm{~h}$, and only a few spherical cells were observed. After $72 \mathrm{~h}$, the cells completely covered the available space in the Petri dish, and created a confluent cell increment. We can thus conclude that the PVA solution did not have any toxic or other effect on the cell adhesion in a normal cell seeding plate. The cells were healthy, and the proliferation was the same as it was in the absence of the PVA solution.
To prevent the polymer's dissolution in water, GDA as a cross-linking agent was used in our systems. GDA solution is a well-known toxic chemical, often utilized in cell science as a fixing agent for different microscopic techniques, as GDA can cross-link the proteins inside the cells $[20,28]$. This reactive behaviour made this chemical a sufficient cross-linker in the polymer science. In the case of GDA, we have observed aggregated spherical cells in both the 24 and $72 \mathrm{~h}$ cultures, which were dead according to the Trypan blue test. The utilized sterilization agent $\left(\mathrm{ClO}_{2}\right)$, did neither harm nor inhibit the attachment and proliferation of the cells $[17,18]$.

\subsection{PVA scaffolds}

After the preparation of the PVA scaffold, a long washing procedure was used in all cases to assure the removal of the unreacted chemicals, the efficiency of which was confirmed by cell experiments. It is evident in literature that remnants of GDA may be present even after washing the polymer matrix [29]. Therefore, the mesh was incubated in cell media for $72 \mathrm{~h}$ in order to remove all the unreacted ingredients from it. After collecting the cell media from the mesh, the cells were mixed with this treated media then seeded in a Petri dish for 24 and $72 \mathrm{~h}$ respectively. The cells obtained a healthy shape, and after a short, $24 \mathrm{~h}$ incubation time adhesion started, and 3 days later all of the cells were attached to the Petri dish, completely covering the available surface (Figure 4). This is indirect evidence that the prepared PVA mesh had no cell-toxic content after preparation and thorough washing.

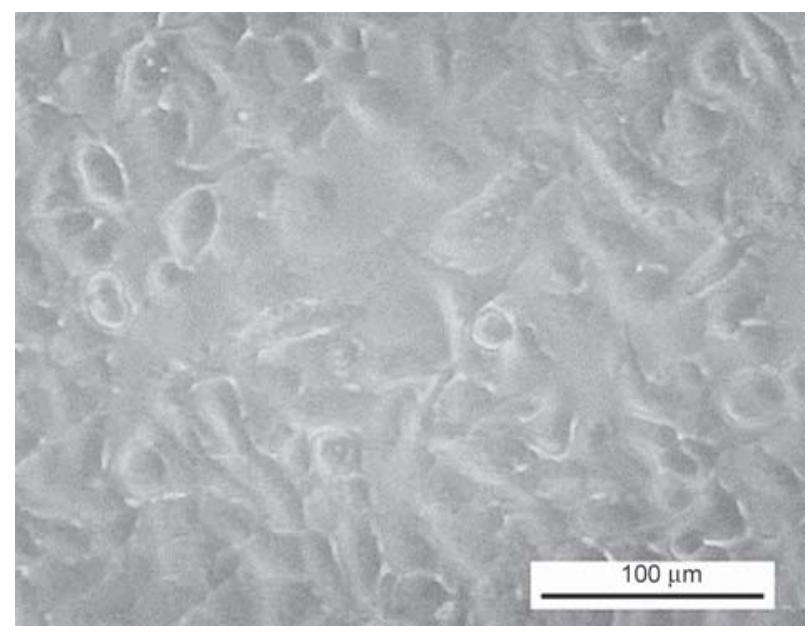

Figure 4. A549 cells after $72 \mathrm{~h}$ incubated in cell media which previously contained a soaked PVA electrospun scaffold. 
The PVA mesh is composed of fibers that can mimic the natural extracellular matrix, which is the scaffold upon cells live and proliferate in vivo. The electrospun PVA-GDA scaffold becomes a chemical gel in the presence of $\mathrm{HCl}$. Further, placing this mesh to the PBS solution, additional physical cross-links are formed in the presence of salts at the ionic strength characteristic to living systems.

In our experiments two different setups were used: The first setup, referred to here as method A, included a washing step with water, while in the other setup, called method B, included an incubation step in cell media. Both in Methods A and B, the cell adhesion onto the PVA mesh was not completely satisfactory. Cells could only attach to the bottom of the Petri dish, however, there they obtained a healthy triangular shape and then proliferated. Although the cells could not attach to the surface of the meshes, the presence of the mesh did not affect the normal cell life.

According to our results, the PVA meshes did not have any toxic effects on the cells; however, it could be observed that the surface of the PVA meshes was not suitable for A549 cells to attach to, since normal cell morphology was not observed. Therefore, we conclude that the surface of the PVA mesh inhibits the adhesion of cells. This property can be useful in some biomedical applications, especially in hernia treatment, where undesirable adhesion of abdominal organs to the implants can elicit serious complications [9].

\subsection{Mechanical tests}

Polypropylene (PP)-based meshes (such as the one used as the control system in our experiments) are widely used in the treatment of abdominal hernia; therefore, their properties have been comprehensively examined and reported in the literature. The type of mesh used as control in our experiments can withstand an approximately $64 \mathrm{~N}$ pulling force before it breaks during a standard uniaxial measurement, which is more than enough to withstand the average $23 \mathrm{kPa}$ pressure arising in the abdomen during coughing or jumping [30] or the general $16 \mathrm{~N} / \mathrm{cm}^{-1}$ in the case of small hernias [31].

The mechanical properties of a PVA mesh and a control PP mesh were investigated in order to predict their performance during implantation in the following in vivo experiments, described in detail in Section 2.7. The breaking point of the electrospun PVA mesh was measured without using stitches to fix it. The gel fibers of our mesh contains $70 \pm 2 \mathrm{w} / \mathrm{w} \%$ PBS. Every hydrogel is capable of changing its swelling degree, depending on the environmental conditions. In wet conditions the gel fibers can swell. In the opposite case the gel fibers can dry out. The kinetics of both processes (swelling and drying) takes much longer time than the duration of mechanical measurements. Therefore, it is reasonable to assume, that there was no volume change during the mechanical study. The maximum extension was $3 \mathrm{~cm}$ in each case, and when the force reached $17 \mathrm{~N}$, the mesh was torn (Figure 5a). As it can be seen on Figure 5a the PVA mesh used in the experiment does not show neither Hookean nor rubber elastic behaviour. This is due to the fact that the PVA samples have a hierarchical structure composed of a mesh of randomly oriented and fused gel fibers. Our knowledge is poor on the mechanical properties of such materials. According to our knowledge understanding the mechanical behaviour of such complex structure has not been worked out. It is worth noting that samples were torn near the clamps pulling them during the measurement.

The intra-abdominal pressure however acts upon the suture line, not just on the mesh itself, therefore, investigating the effects of stitches on the mechanical properties is essential [32]. During the mechanical measurements, sutures were used to fixate the samples. Both samples (PVA and PP) were torn mildly at a $5 \mathrm{~N}$ load, due to the acted force being divided unequally among each stich, hence the sample torn itself until the load was evenly distributed. A typical example of how applied force acts upon the PVA samples can be seen on Figure 5b. During force exertion the PP mesh behaved similarly, however, there was no observable tearing or damage, only slight deformation was observed (Figure $5 \mathrm{~d}$ ).

Figure $5 \mathrm{c}$ shows the PVA scaffold after reaching the critical load at $13.85 \mathrm{~N}$, where the suture line was ripped out of the sample. In both samples, the ripping suture line formed the same zig-zag pattern, marking the needle penetration points 'valleys' during the suturing of the samples (Figure $5 \mathrm{~b}$ and $5 \mathrm{~d}$ ). PP meshes were significantly stronger, hence their breaking point reached $90 \mathrm{~N}$, where not the mesh, but the suture material was actually torn, however at loads this high (as it can be seen on Figure 5e) the mesh has already deformed permanently. It should be noted that our result on the PP mesh is somewhat different from 


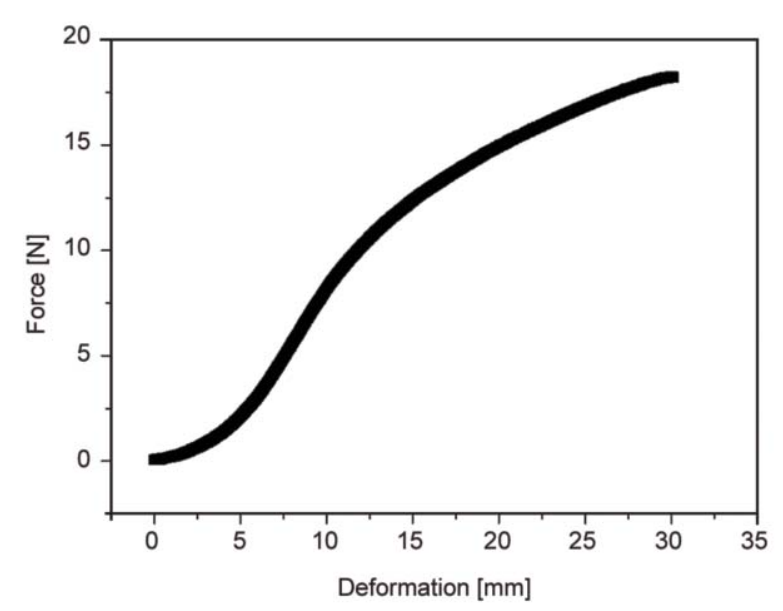

a)

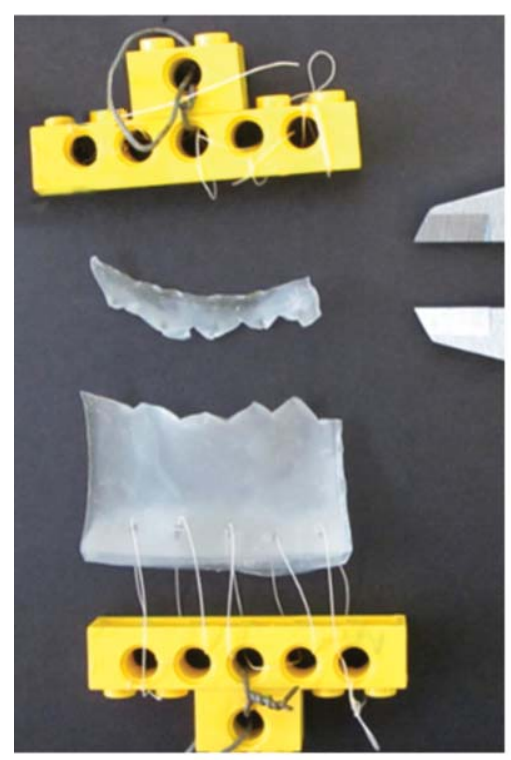

c)

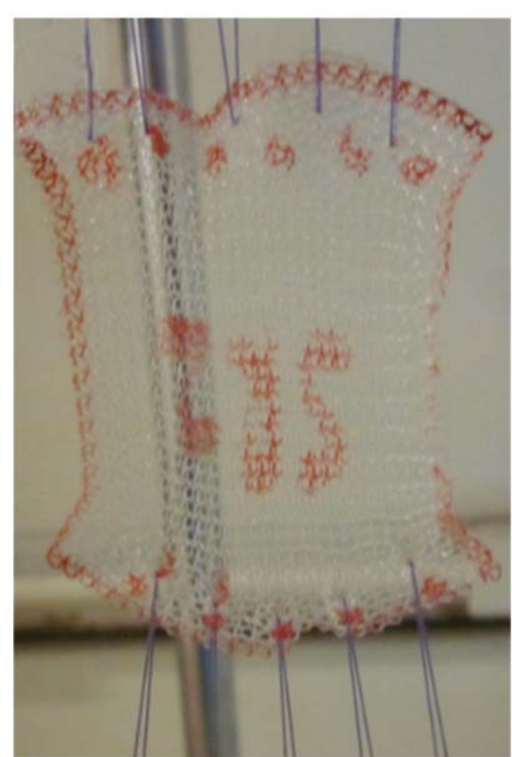

d)
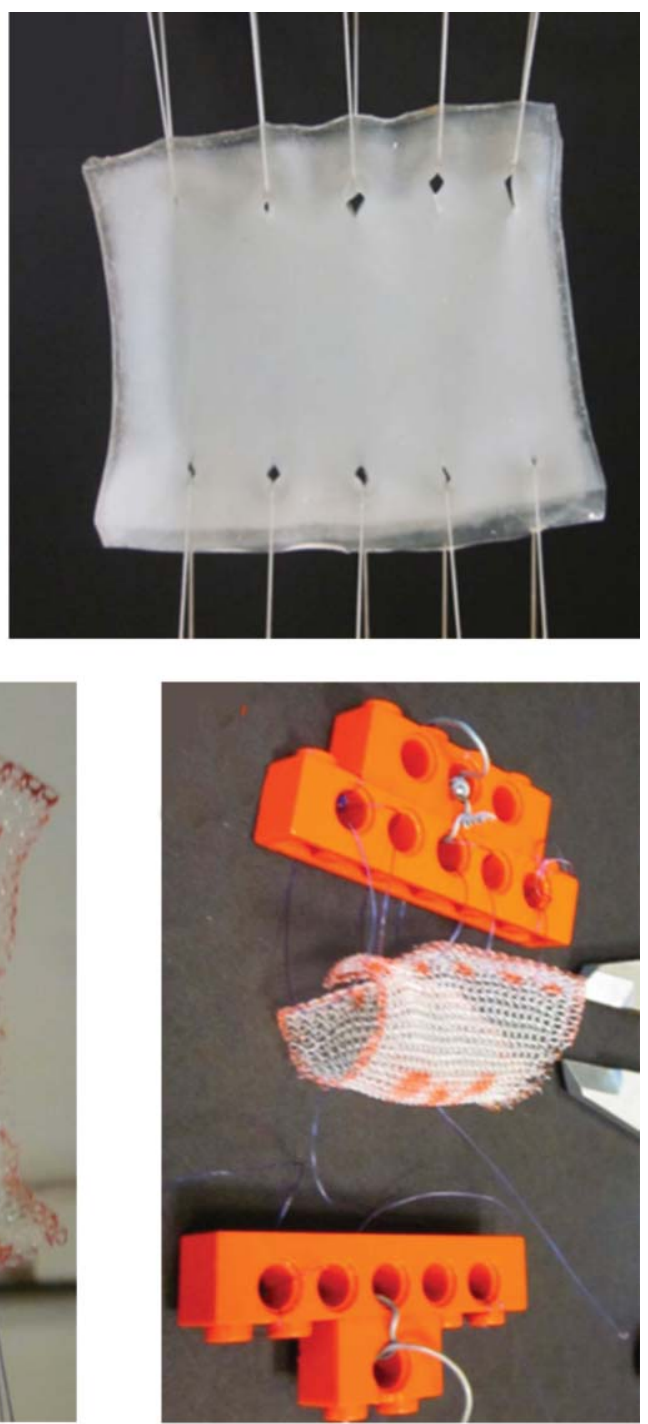

e)

Figure 5. Tensile mechanical curve of PVA mesh in uni-axial measurement (a), effects of $5 \mathrm{~N}$ load (b) and a torn PVA mesh (c), the defects on the PP mesh at a load of $13 \mathrm{~N}$ (d) and the deformed PP mesh (e) (calliper set to $1 \mathrm{~cm}$ ).

that measured by Li et al. [30], probably because of the different measurement setup and sample size. In conclusion, we found that the cross-linked PVA mesh is suitable for in vivo experiments, as it can withstand the conditions of mesh implantation and the abdominal pressure after surgery.

\subsection{Results of animal studies}

Based on our promising results from cell and mechanical tests, the biomechanical features and the extent of adhesion, formation of PVA meshes were investigated in animal studies, as well. The purpose of the animal studies was to monitor the PVA mesh after its intra-peritoneal placement either on an intact peritoneum, or on a created abdominal wall defect, simulating laparoscopic ventral hernia repair, and also to evaluate host tissue response. The mesh was adequate for surgical procedure, i.e., easy to suture, and, as a soft and flexible material, it adapted well to abdominal tissues. All meshes used held their strength and structure. There was no post-implant mortality, and no signs of serious infection or rejection. Mesh incorporation was complete in all animals; only minimal adhesion was observed in three rats. In contrast, dense adhesions were developed on the majority of the PP suture line (13 rats from the 27 animals with score 1) (Figure 6. and Table 1). Compared to the PVA mesh, the total score of adhesions was significantly higher $(p<0.05)$ (Table 1.). PP is well known for its good tissue integration profile, but it induces severe adhesions [33]. In three cases, seroma and slight perigraft reactions (inflammations) occurred. By contrast to PP, the PVA mesh did not induce significant adhesion. Formation of the neoperitoneum 


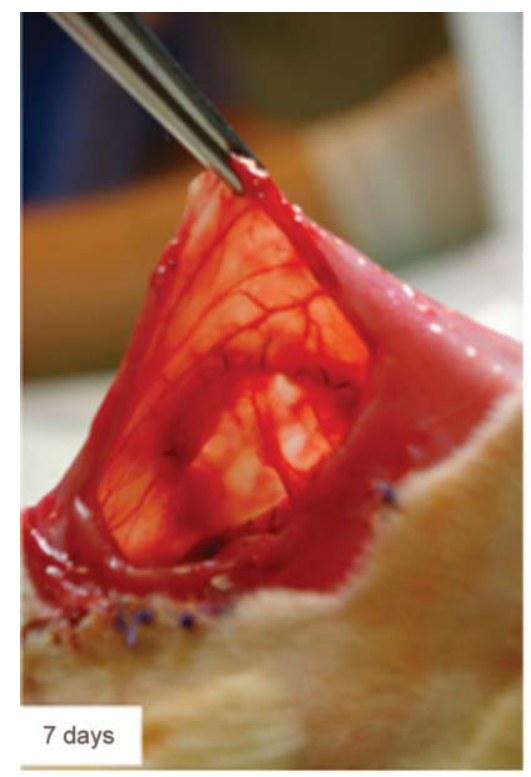

a)

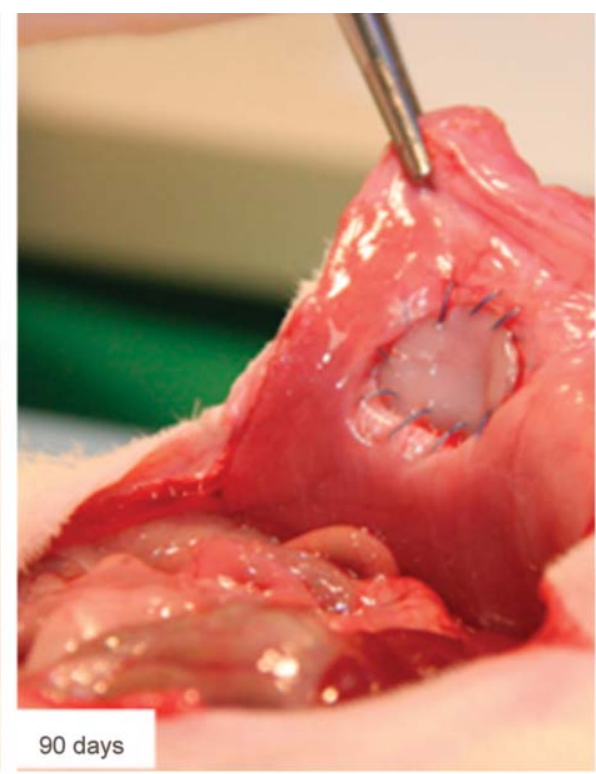

b)

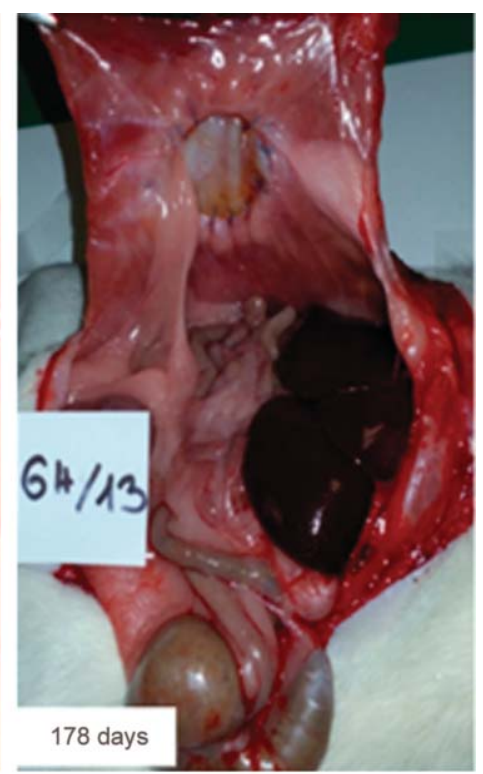

c)

Figure 6. PVA mesh incorporation - macroscopic findings (samples at a) 7, b) 90 and c) 178 days).

Table 1. Overall diamond scale scores throughout the in vivo animal experiments.

\begin{tabular}{|c|c|c|}
\hline Score & $\begin{array}{c}\text { Extent } \\
{[\%]}\end{array}$ & Animal \\
\hline 0 & 0 & 3 \\
\hline 1 & $<25$ & 27 \\
\hline 2 & $25-50$ & 8 \\
\hline 3 & $50-75$ & 4 \\
\hline 4 & $>75$ & 0 \\
\hline
\end{tabular}

was complete in the adhesion-free areas. In four animals we found adhesion formation between $50-75 \%$ with other organs (liver, bowel), these animals were given 3 point according to the Diamond scale.

Our results showed firm integration of the mesh structure within the surrounding tissue after 3 and 6 months, with mild chronic inflammatory reaction, however, the long-term behaviour of intra-abdominally placed PVA meshes should still be determined.

Histology performed on samples from groups II and III on termination days 7 and 14 showed similar results, therefore they will be discussed together. PVA meshes were all well integrated into the surrounding tissue (Figure 7a) with a physiological tissue response (foreign body type reaction). There was granulation tissue formation with variable number of neutrophils and scattered giant cells adjacent to the PVA meshes. The meshes were typically intact with attached neutrophils, histiocytes, and giant cells on their surface. Some meshes were invaded by inflammatory cells in one or two places close to the edge.
Samples from groups II and III on the $28^{\text {th }}$ termination day reviled diminished granulation tissue, which was partially replaced by mild fibrosis, and thus the rate of healing process matched that of a physiologic one. Meshes were covered mainly by histiocytes and/or giant cells, and some meshes were invaded by a thin bundle of granulation tissue or giant cells (Figure 7b). However invasion into the new tissue was possible at the sample's torn segment, where the layers of the PVA meshes were slightly separated. In our opinion, this cannot be considered as an indication of biodegradation, but only of a physical degradation. Samples from group I showed very similar histological features, with an even milder granulation and/or fibrotic tissue formation around the meshes. It is important to mention however, that group I animals underwent surgery of moderate level, as compared to group II and III animals, where a small portion of the abdominal muscle wall was cut out (artificial defect). Samples from control group IV showed no alterations or only foreign body type giant cell reaction around the sutures.

Samples from both groups II and III on the $90^{\text {th }}$ and $178^{\text {th }}$ termination days showed mild fibrosis surrounding the meshes with scattered histiocytes and giant cells. The meshes were either intact, or contained some histiocytes/giant cells, groups of ghost cells (dead cells), or fibrotic tissue bundles (Figure 7c), signifying the last phase of the physiologic healing process taking place, when the $\backslash$ implant incorporates into the surrounding tissue. Degradation 

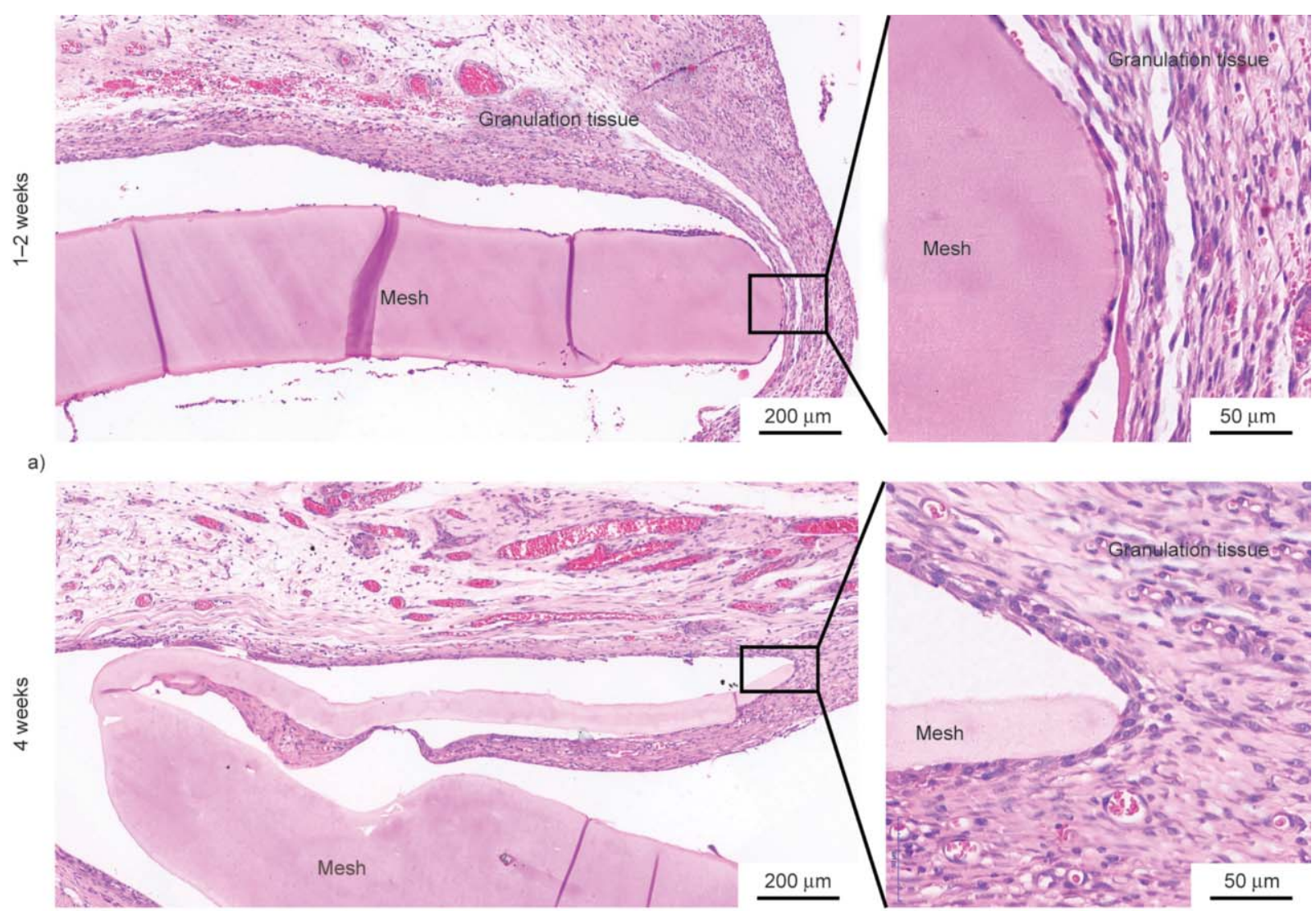

b)

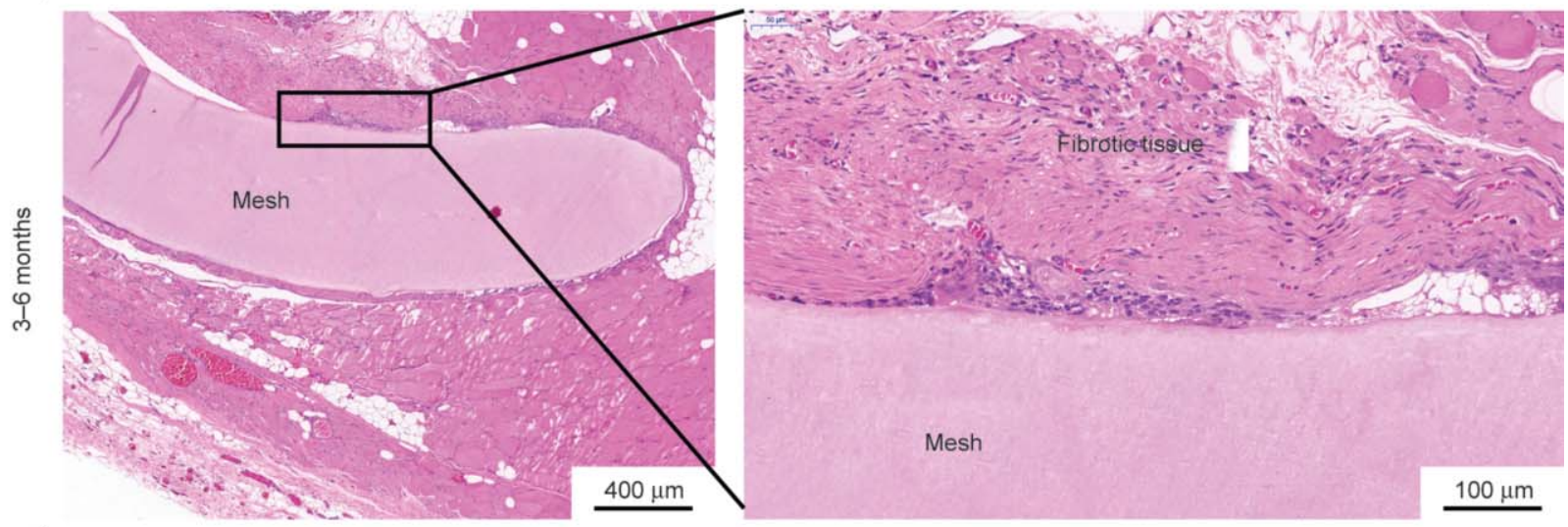

c)

Figure 7. A typical picture of a PVA mesh after 1-2 weeks (a), 4 weeks (b) and 3-6 months (c) after implantation.

of the meshes was not observable by macroscopic observation. Nor by histopathologic examination. The microstructure of the PVA meshes implanted in the animals (due to the relatively small pore sizes) would normally promote bacterial adhesion and biofilm formation, which leading to serious inflammatory response, a phenomenon reported by Nisticó et al. [5]. On the contrary in our case no histopathological signs were found suggesting such complications.

\section{Conclusions}

Preparing an absorbable, polymer-based hernia mesh with specified properties, such as biocompatibility and integrity, flexibility, and suturability is still a challenging task. However, the electrospun PVA mesh introduced in this paper satisfies all of these criteria. Furthermore, it was also found that this mesh was easily integrated into the surrounding tissue upon implantation without serious inflammatory reaction. The biocompatibility of electrospun PVA meshes are carefully and clearly demonstrated in this work. The mesh has an outstanding suturability as well as it provides a good platform for native tissue to get attached to. Complications in mesh-based hernia repair arise from adhesion of viscera to the implanted mesh, which, in our case, only happened in 3 out of 45 animals. Fur- 
ther, the PVA scaffolds prevent adhesion formation to the intra-abdominal structures. Our results strongly suggest that electrospun PVA mesh could be a potential future surgical mesh.

\section{Acknowledgements}

This research was supported by OTKA K 105523, NKFI FK 124147 and supported by the ÚNKP-17-3-III-SE-18 New National Excellence Program of the Ministry of Human Capacities.

\section{References}

[1] Flum D. R., Horvath K., Koepsell T.: Have outcomes of incisional hernia repair improved with time? A population-based analysis. Annals of Surgery, 237, 129135 (2003).

https://doi.org/10.1097/00000658-200301000-00018

[2] Kingsnorth A., LeBlanc K.: Hernias: Inguinal and incisional. Lancet, 362, 1561-1571 (2003). https://doi.org/10.1016/S0140-6736(03)14746-0

[3] Rudmik L. R., Schieman C., Dixon E., Debru E.: Laparoscopic incisional hernia repair: A review of the literature. Hernia, 10, 110-119 (2006).

https://doi.org/10.1007/s10029-006-0066-6

[4] Goodney P. P., Birkmeyer C. M., Birkmeyer J. D.: Short-term outcomes of laparoscopic and open ventral hernia repair: A meta-analysis. Archives of Surgery, 137, 1161-1165 (2002).

https://doi.org/10.1001/archsurg.137.10.1161

[5] Nisticò R., Magnacca G., Martorana S.: Surface science in hernioplasty: The role of plasma treatments. Applied Surface Science, 419, 860-868 (2017).

https://doi.org/10.1016/j.apsusc.2017.05.121

[6] Hjort H., Mathisen T., Alves A., Clermont G., Boutrand J. P.: Three-year results from a preclinical implantation study of a long-term resorbable surgical mesh with time-dependent mechanical characteristics. Hernia, 16, 191-197 (2012).

https://doi.org/10.1007/s10029-011-0885-y

[7] Huang Z-M., Zhang Y-Z., Kotaki M., Ramakrishna S.: A review on polymer nanofibers by electrospinning and their applications in nanocomposites. Composites Science and Technology, 63, 2223-2253 (2003). https://doi.org/10.1016/S0266-3538(03)00178-7

[8] Caló E., Khutoryanskiy V. V.: Biomedical applications of hydrogels: A review of patents and commercial products. European Polymer Journal, 65, 252-267 (2015). https://doi.org/10.1016/j.eurpolymj.2014.11.024

[9] Renz B. W., Leitner K., Odermatt E., Worthley D. L., Angele M. K., Jauch K-W., Lang R. A.: PVA gel as a potential adhesion barrier: A safety study in a large animal model of intestinal surgery. Langenbeck's Archives of Surgery, 399, 349-357 (2014).

https://doi.org/10.1007/s00423-013-1159-1
[10] Kim C. H., Khil M. S., Kim H. Y., Lee H. U., Jahng K. Y.: An improved hydrophilicity via electrospinning for enhanced cell attachment and proliferation. Journal of Biomedical Materials Research Part B: Applied Biomaterials, 78B, 283-(2006).

https://doi.org/10.1002/jbm.b.30484

[11] Gupta S., Webster T. J., Sinha A.: Evolution of PVA gels prepared without crosslinking agents as a cell adhesive surface. Journal of Materials Science: Materials in Medicine, 22, 1763-1772 (2011). https://doi.org/10.1007/s10856-011-4343-2

[12] Kenawy E-R., Abdel-Hay F. I., El-Newehy M. H., Wnek G. E.: Controlled release of ketoprofen from electrospun poly(vinyl alcohol) nanofibers. Materials Science and Engineering: A, 459, 390-396 (2007).

https://doi.org/10.1016/j.msea.2007.01.039

[13] Cui Z., Zheng Z., Lin L., Si J., Wang Q., Peng X., Chen $\mathrm{W}$.: Electrospinning and crosslinking of polyvinyl alcohol/chitosan composite nanofiber for transdermal drug delivery. Advances in Polymer Technology, in press (2018). https://doi.org/10.1002/adv.21850

[14] Wang M., Roy A. K., Webster T. J.: Development of chitosan/poly(vinyl alcohol) electrospun nanofibers for infection related wound healing. Frontiers in Physiology, 7, 683/1-683/3 (2017). https://doi.org/10.3389/fphys.2016.00683

[15] Zhang T., Zhang Z., Hu W., Ji Z., Gu N.: Preparation of poly(vinyl alcohol) modified polypropylene mesh and its antiadhesion efficacy in experimental hernia repair. Colloids and Surfaces A: Physicochemical and Engineering Aspects, 500, 10-16 (2016). https://doi.org/10.1016/j.colsurfa.2016.04.010

[16] Molnár K., Juriga D., Nagy P. M., Sinkó K., Jedlovszky-Hajdu A., Zrínyi M.: Electrospun poly(aspartic acid) gel scaffolds for artificial extracellular matrix. Polymer International, 63, 1608-1615 (2014). https://doi.org/10.1002/pi.4720

[17] Noszticzius Z., Wittmann M., Kály-Kullai K., Beregvári Z., Kiss I., Rosivall L., Szegedi J.: Chlorine dioxide is a size-selective antimicrobial agent. PLoS ONE, 8, e79157/1-e79157/10 (2013). https://doi.org/10.1371/journal.pone.0079157

[18] Singh N., Singh R. K., Bhunia A. K., Stroshine R. L.: Efficacy of chlorine dioxide, ozone, and thyme essential oil or a sequential washing in killing Escherichia coli O157:H7 on lettuce and baby carrots. LWT - Food Science and Technology, 35, 720-729 (2002).

https://doi.org/10.1006/fstl.2002.0933

[19] Matthews B. D., Mostafa G., Carbonell A. M., Joels C. S., Kercher K. W., Austin C., Norton H. J., Heniford B. T.: Evaluation of adhesion formation and host tissue response to intra-abdominal polytetrafluoroethylene mesh and composite prosthetic mesh. Journal of Surgical Research, 123, 227-234 (2005). https://doi.org/10.1016/j.jss.2004.08.012 
[20] Shalumon K. T., Binulal N. S., Selvamurugan N., Nair S. V., Menon D., Furuike T., Tamura H., Jayakumar R.: Electrospinning of carboxymethyl chitin/poly(vinyl alcohol) nanofibrous scaffolds for tissue engineering applications. Carbohydrate Polymers, 77, 863-869 (2009). https://doi.org/10.1016/j.carbpol.2009.03.009

[21] Zhang Y. Z., Venugopal J., Huang Z-M., Lim C. T., Ramakrishna S.: Crosslinking of the electrospun gelatin nanofibers. Polymer, 47, 2911-2917 (2006).

https://doi.org/10.1016/j.polymer.2006.02.046

[22] Tang C., Saquing C. D., Harding J. R., Khan S. A.: In situ cross-linking of electrospun poly(vinyl alcohol) nanofibers. Macromolecules, 43, 630-637 (2010). https://doi.org/10.1021/ma902269p

[23] Lu W., Ma M., Xu H., Zhang B., Cao X., Guo Y.: Gelatin nanofibers prepared by spiral-electrospinning and cross-linked by vapor and liquid-phase glutaraldehyde. Materials Letters, 140, 1-4 (2015).

https://doi.org/10.1016/j.matlet.2014.10.146

[24] Molnár K., Jedlovszky-Hajdu A., Zrínyi M., Jiang S., Agarwal S.: Poly(amino acid)-based gel fibers with $\mathrm{pH}$ responsivity by coaxial reactive electrospinning. Macromolecular Rapid Communications, 38, 1700147/11700147/5 (2017).

https://doi.org/10.1002/marc.201700147

[25] Salehi R., Irani M., Rashidi M-R., Aroujalian A., Raisi A., Eskandani M., Haririan I., Davaran S.: Stimuli-responsive nanofibers prepared from poly $(N$-isopropylacrylamide-acrylamide-vinylpyrrolidone) by electrospinning as an anticancer drug delivery. Designed Monomers and Polymers, 16, 515-527 (2013). https://doi.org/10.1080/15685551.2013.771303

[26] Aboutalebi Anaraki N., Roshanfekr Rad L., Irani M., Haririan I.: Fabrication of PLA/PEG/MWCNT electrospun nanofibrous scaffolds for anticancer drug delivery. Journal of Applied Polymer Science, 132, 41286/141286/9 (2015).

https://doi.org/10.1002/app.41286
[27] Dubey P., Gopinath P.: Fabrication of electrospun poly(ethylene oxide)-poly(capro lactone) composite nanofibers for co-delivery of niclosamide and silver nanoparticles exhibits enhanced anti-cancer effects in vitro. Journal of Materials Chemistry B, 4, 726-742 (2016).

https://doi.org/10.1039/C5TB02351C

[28] Takigawa T., Endo Y.: Effects of glutaraldehyde exposure on human health. Journal of Occupational Health, 48, 75-87 (2006). https://doi.org/10.1539/joh.48.75

[29] Mansur H. S., Sadahira C. M., Souza A. N., Mansur A. A. P.: FTIR spectroscopy characterization of poly (vinyl alcohol) hydrogel with different hydrolysis degree and chemically crosslinked with glutaraldehyde. Materials Science and Engineering: C, 28, 539-548 (2008). https://doi.org/10.1016/j.msec.2007.10.088

[30] Li X., Kruger J. A., Jor J. W. Y., Wong V., Dietz H. P., Nash M. P., Nielsen P. M.: Characterizing the ex vivo mechanical properties of synthetic polypropylene surgical mesh. Journal of the Mechanical Behavior of Biomedical Materials, 37, 48-55 (2014).

https://doi.org/10.1016/j.jmbbm.2014.05.005

[31] Kalaba S., Gerhard E., Winder J. S., Pauli E. M., Haluck R. S., Yang J.: Design strategies and applications of biomaterials and devices for hernia repair. Bioactive Materials, 1, 2-17 (2016).

http://inkinghub.elsevier.com/retrieve/pii/S2452199X16300093

[32] Chakroff J., Kayuha D., Henderson M., Johnson J.: Development and characterization of novel electrospun meshes for hernia repair. SOJ Materials Science and Engineering, 3, 1-9 (2015). https://doi.org/10.15226/sojmse.2015.00114

[33] Halm J. A., de Wall L. L., Steyerberg E. W., Jeekel J., Lange J. F.: Intraperitoneal polypropylene mesh hernia repair complicates subsequent abdominal surgery. World Journal of Surgery, 31, 423-429 (2007). https://doi.org/10.1007/s00268-006-0317-9 